\title{
Energy efficiency of cooperative D2D communications underlaying LTE-A networks
}

\author{
Xiaoying Zhang, Ahmed Khwaja, Muhammad Naeem, and Alagan Anpalagan* \\ Dept. of Electrical and Computer Engineering, Ryerson University, Canada
}

\begin{abstract}
Device-to-device (D2D) communications underlaying LTE-A networks is expected to bring significant benefits for resource utilization and energy efficiency (EE) improvement of user equipment (UE). However, the allocation of radio and power resources to D2D communications needs elaborate coordination, because of the interference between D2D communications and cellular communications. In this paper, we propose an energy-efficient cooperative D2D communication (EECD2D) technique using a power allocation algorithm, aiming at maximizing EE introduced by D2D communications in LET-A networks. Specifically, we define four D2D and cellular combinations based on distances, and analyze average EE of EECD2D and that of cooperative D2D communications without optimization. Results show that average EE of our algorithm is much higher than that without optimization, and closer D2D cooperators and distant cellular UEs whose uplink resource is reused, achieve highest average energy efficiency.
\end{abstract}

\section{Introduction}

To incorporate D2D communications into LTE-A networks, new features and functionalities related to $\mathrm{D} 2 \mathrm{D}$ communications need to be introduced into the existing LTE-A networks. Typical LTE-A networks supporting D2D communications involve proximity-based service (ProSe) Function and ProSe Application Server, which provide functions and service for D2D discovery and D2D communications. Moreover, resource management must be considered to improve the resource utilization and network performance. Generally, resource management includes three aspects: mode selection, channel assignment and power allocation. Effective resource management can not only improve the radio resource utilization, but also energy efficiency (EE) of the network. Researches [1-4] mainly focused on how to maximize spectrum efficiency and network throughput, while ignoring the energy consumption of user equipment. In practical implementation, user equipment are typically handheld devices with limited battery life and can quickly run out of battery without proper energy management. In this paper, we aim at maximizing the EE through power allocation, and analyzing EE of different D2D cooperator selection and channel assignment methods. In [5], an analytical model of EE for different sharing modes is proposed. Then, the energy-efficient uplink resource sharing problem is formulated as a nontransferable coalition formation game to address the joint issue of the mode selection, uplink reusing allocation, and power management. This system

*Corresponding author: alagan@ee.ryerson.ca 
model also assumes that a D2D pair shares the same uplink resources with multiple selected cellular users. In [6], the joint mode selection and spectrum sharing is also modeled as a coalition formation game and a coalition formation algorithm is proposed to jointly solve the mode selection and spectrum sharing in a D2D system. Unlike [5], the transmit power of each user is fixed. Specifically, they assume that one cellular user could share spectrum with multiple D2D pairs, and the objective is to improve the EE of all the wireless users. To the best of our knowledge, D2D communications underlaying cellular network in recent work are performed when the receiver of data packets are within the D2D communication range or can be reached within two hops, and thus it can reduce the energy consumption through direct communications. In our algorithm, the D2D communication is used to forward data packets to the eNB and then to the destination user equipment. It helps to complete long distance cellular communications if the transmitter is not able to do it. The D2D cooperator can be selected in different methods, i.e. distances to the D2D requester (also the transmitter of a D2D pair). Results show that different distance levels have different influence on EE introduced by D2D communications.

Unlike other research work that aims at maximizing system EE or individual link EE only, our goal is to maximize total EE introduced by D2D communications using power allocation. Here, EE is the sum of EE of D2D link and EE of cellular link from D2D cooperator (also the receiver of D2D pairs) to the eNB. User equipment whose battery is below the threshold or channel condition is bad will send a D2D request to the eNB for setting up D2D communications. Since EE maximization does not guarantee the longest lifetime which is the time when the first user equipment runs out of its battery, our algorithm can protect user equipment's battery life. The rest of this paper is organized as follows. In Section II, we describe network model and assumptions. Section III presents detailed description of the problem formulation. Section IV shows the simulation results and performance comparison. Finally, Section V concludes the paper.

\section{Network Model and Assumptions}

In this paper, we consider a single cell scenario, where one eNB is located at the center and some user equipment is randomly distributed. CUEs are cellular users which directly communicate with the eNB, and DUEs are D2D users between which the direct communication can be supported without going through the eNB. Each CUE is allocated with an orthogonal resource block, and thus, there is no co-channel interference between CUEs. As shown in figure 1, the color of each CUE's uplink channel is different, which presents different frequency. There exist also three D2D pairs in the network. We assume that eNB has the CSI (channel state information) of all the involved links, so that it can allocate the available radio resources. The eNB can allocate either orthogonal or non-orthogonal radio resources to DUEs. If the resources occupied by the DUEs are orthogonal to those allocated to the CUEs, there is no interference to each other and the analysis is simpler. However, this mode leads to low resource utilization. Generally speaking, there are basically three communication modes for cellular systems supporting D2D:

- Cellular mode: User equipment communicates as conventional CUEs through eNB.

- Orthogonal Sharing mode (OS): DUEs and the CUE take the exclusive resources and there is no interference between them.

- Non-Orthogonal Sharing mode (NOS): The same resources are reused by the CUE and DUEs, which causes interference to each other.

Form the spectrum efficiency perspective, NOS mode is used for DUE resource allocation in this paper. In figure $1, \mathrm{D} 2 \mathrm{D}$ pair $D P_{1}$ reuses the uplink channel of $C_{4}, \mathrm{D} 2 \mathrm{D}$ pair $D P_{2}$ reuses the uplink channel of $C_{6}$, and $\mathrm{D} 2 \mathrm{D}$ pair $D P_{3}$ reuses the uplink channel of $C_{l}$. 
We assume that there is a set $C=\left\{1, \cdots, C_{M}\right\}$ of uplinks of CUEs occupying $M$ orthogonal channels in the cell and a set $D=\left\{1, \cdots, D_{N}\right\}$ of D2D links which transmit data using the same set of channels, where $M \geq N$. We also assume that each D2D pair could only reuse the uplink channel of one CUE and each uplink channel of CUE could be reused by only one DUE pair. The reused channel for each D2D pair has been pre-defined in the system. $P_{D_{i}}, P_{R_{i}}, P_{C_{i}}$ are the transmit power of any D2D transmitter $D_{i}$, D2D receiver $R_{i}$, and any CUE $C_{i}$, respectively. $P_{\text {cir }}$ is the average circuit power consumed by D2D communications. Without loss of generality, we assume that all UEs have the same constant circuit power consumption. In addition, the propagation channel model contains path loss and Rayleigh fading.

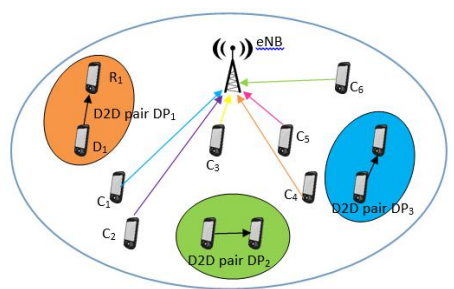

Fig. 1. Illustration of uplink channel reuse for D2D communications underlying cellular networks.

In figure $1, D P_{1}$ reuses the uplink channel of $C_{4}$. Let $L_{c}, L_{d}$ and $L_{r}$ be the distance between $C_{4}$ and $R_{l}$, distance between $D_{l}$ and $R_{l}$, and distance between $R_{l}$ to the eNB (which is also shown in figure 2). $N_{0}$ is the average power of additive Gaussian noise. All links are assumed to experience independent block fading. Hence, the instantaneous channel gain of the interference link between $C_{4}$ and the $R_{l}$ can be expressed as

$$
\mathrm{H} \mathrm{D}^{\mathrm{C}}=\varphi \mathrm{L} \mathrm{c} \mathrm{c}^{-\alpha}
$$

where $\alpha$ is the path loss exponent, $\varphi$ denotes the channel fading component, where the Rayleigh fading coefficient follows an exponential distribution with unit mean. Similarly, we can express the channel gain between $R_{l}$ and the eNB as $H_{B}{ }^{R}$, the channel gain between the D2D pair as $H_{D}$.

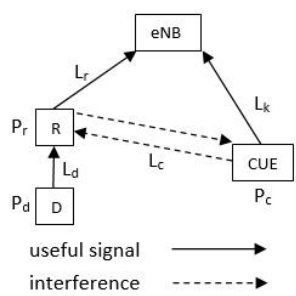

Fig. 2. Interference Model of D2D communications in NOS Mode.

\section{Problem Formulation}

Unlike D2D communication used for direct communication between source DUE and destination DUE, our D2D communication is used for cooperation. The D2D requester (denoted as $D$ ) refers to any CUE, i.e. a CUE whose battery is below the battery threshold, that needs to establish D2D communication. The D2D cooperator (denoted as $R$ ) helps to forward D2D requester's data to the eNB, and the eNB forward data to the destination CUE as in traditional cellular communications. Due to the simultaneous transmission, the DUE and the CUE (denoted as $C$ ) whose uplink channel is reused cause interference to each other. figure 2 shows the interference model of one D2D pair and a CUE. The received signal-to-interference-plus-noise ratio (SINR) of the receiver $R_{i}$ on the uplink of $C_{i}$ is given as follows: SINR of D2D pair $\left(D_{i}, R_{i}\right)$ is

$$
\gamma_{D_{i}}{ }^{C}=\frac{P_{D_{i}} H_{D_{i}}}{P_{C_{i}} H_{D_{i}}{ }^{C_{i}}+N_{0}}=\frac{P_{D_{i}} \varphi_{i} L_{d_{i}}{ }^{-\alpha}}{P_{C_{i}} \varphi_{i} L_{c_{i}}{ }^{-\alpha}+N_{0}}
$$


The received SINR for D2D receiver $R_{i}$ at the eNB is given as follows:

$$
\gamma_{B}{ }^{R_{i}}=\frac{P_{R_{i}} H_{B}{ }^{R_{i}}}{N_{0}}=\frac{P_{R_{i}} \varphi_{i} L_{r_{i}}{ }^{-\alpha}}{N_{0}}
$$

In this paper, for conciseness, we consider a unit bandwidth uplink channel for CUEs. Then, the throughput of $D_{i}$ and $R_{i}$ can be obtained, respectively, as follows: Thoughput of $D_{i}$ is

$$
R_{D_{i}}=\log _{2}\left(1+\gamma_{D_{i}}^{C}\right)
$$

Thoughput of $R_{i}$ is

$$
R_{R_{i}}=\log _{2}\left(1+\gamma_{B}^{R_{i}}\right)
$$

EE of $D_{i}$ link is

$$
E E_{D_{i}}=\frac{\log _{2}\left(1+\gamma_{D_{i}}^{C}\right)}{P_{D_{i}}+P_{c i r}}=\frac{\log _{2}\left(1+\frac{P_{D_{i}} \varphi_{i} L_{d_{i}}{ }^{-\alpha}}{P_{C_{i} \varphi_{i} L_{c_{i}}{ }^{-\alpha}+N_{0}}}\right)}{P_{D_{i}}+P_{c i r}}
$$

EE of $R_{i}$ link is

$$
E E_{R_{i}}=\frac{\log _{2}\left(1+\gamma_{B}^{R_{i}}\right)}{P_{R_{i}}+P_{c i r}}=\frac{\log _{2}\left(1+\frac{P_{R_{i}} \varphi_{i} L_{r_{i}}-\alpha}{N_{0}}\right)}{P_{R_{i}}+P_{c i r}}
$$

We define the individual EE as the sum of the EE of D2D link and EE of the cellular link from $R_{i}$ to eNB:

$$
\begin{aligned}
& E E_{i}=E E_{D_{i}}+E E_{R_{i}}=\frac{\log _{2}\left(1+\frac{P_{D_{i}} \varphi_{i} L_{d_{i}}-\alpha}{P_{C_{i}} \varphi_{i} L_{c_{i}}{ }^{-\alpha}+N_{0}}\right)}{P_{D_{i}}+P_{c i r}}+ \\
& \frac{\log _{2}\left(1+\frac{P_{R_{i}} \varphi_{i} L_{r_{i}}-\alpha}{N_{0}}\right)}{P_{R_{i}}+P_{c i r}}
\end{aligned}
$$

For cellular networks supporting $N$ D2D pairs, the total EE of all D2D communications in the network is given below:

The EE optimization function can be formulated as

$$
\begin{aligned}
\sum_{i=1}^{N} E E_{i}=\sum_{i=1}^{N} \frac{\log _{2}\left(1+\frac{P_{D_{i} \varphi_{i} L_{d_{i}}}-\alpha}{P_{C_{i} \varphi_{i} L_{c_{i}}{ }^{-\alpha}+N_{0}}}\right)}{P_{D_{i}}+P_{\text {cir }}}+ \\
\sum_{i=1}^{N} \frac{\log _{2}\left(1+\frac{P_{R_{i}} \varphi_{i} L_{r_{i}}-\alpha}{N_{0}}\right)}{P_{R_{i}}+P_{c i r}}
\end{aligned}
$$

Subject to

$$
\max \sum_{i=1}^{N} E E_{i}
$$

$$
\begin{gathered}
P_{D_{i}} \leq P_{D_{\max }}, \forall i \\
P_{R_{i}} \leq P_{C_{\max }}, \forall i \\
P_{C_{i}} \leq P_{C_{\max }}, \forall i \\
\frac{P_{R_{i}} \varphi_{i} L_{r_{i}}{ }^{-\alpha}}{N_{0}} \geq \gamma_{t h}, \forall i \\
\frac{P_{C_{i}} \varphi_{i} L_{k_{i}}{ }^{-\alpha}}{P_{D_{i}} \varphi_{i} L_{c_{i}}{ }^{-\alpha}+N_{0}} \geq \gamma_{t h}, \forall i
\end{gathered}
$$

$P D_{\max }$ and $P C_{\max }$ denote the maximal transmit power of DUEs and CUEs, respectively. $\gamma_{\text {th }}$ is the SINR threshold. Constraints (11), (12), (13) indicate that the transmit power of DUEs and CUEs cannot exceed their maximum values. Constraint (14) and (15) denote that 
the SINR threshold of CUEs should be guaranteed. By solving the objective function in (10), EE introduced by cooperative D2D communication is maximized and transmit power of all related user equipment is adjusted.

\section{Performance Evaluation}

In this section, we provide several simulation results to evaluate the performance of EECD2D algorithm, and compare it with the performance of cooperative D2D communications without EE optimization. A single circular cell environment is considered. 100 users are randomly placed in the cell, where the eNB is at the center. The results are averaged over 100 realizations. Main simulation parameters are presented in table 1 . Since the optimization problem is a complex non-linear problem with large number of variables, we use the Matlab Genetic Algorithm Toolbox to solve it.

Table 1. Simulation Parameters.

\begin{tabular}{|c|c|}
\hline Parameter & Value \\
\hline Cell Radius & $500 \mathrm{~m}$ \\
\hline D2D Communication Range & $50 \mathrm{~m}$ \\
\hline Number of total UEs & 100 \\
\hline Number of D2D Pairs & $5,10,15,20,25$ \\
\hline Channel Fading Coefficient $(\varphi)$ & exponential distribution with unit mean \\
\hline Path Loss Exponent $(\alpha)$ & 4 \\
\hline Maximum UE Tx power $\left(P P_{\max }, P C_{\max }\right)$ & $0.25 \mathrm{~W}$ \\
\hline Circuit Power $\left(P_{c i r}\right)$ & $0.05 \mathrm{~W}$ \\
\hline Noise Power $\left(N_{0}\right)$ & $10^{-13} \mathrm{~W}$ \\
\hline SINR threshold $\left(\gamma_{t h}\right)$ & $10 \mathrm{~dB}$ \\
\hline
\end{tabular}

In order to analyze the performance affected by different locations of D2D and CUEs, we simulate four types of scenarios:

- Type 1 is close D2D and far CUE. Distance between each D2D pair is within 25m, and distance between each D2D pair and the CUE whose uplink resource is reused is larger than $300 \mathrm{~m}$.

- Type 2 is far D2D and far CUE. Distance between each D2D pair is larger than 25m, and distance between each D2D pair and the CUE whose uplink resource is reused is larger than $300 \mathrm{~m}$.

- Type 3 is close D2D and close CUE. Distance between each D2D pair is within 25m, and distance between each D2D pair and the CUE whose uplink resource is reused is within $300 \mathrm{~m}$.

- Type 4 is far D2D and close CUE. Distance between each D2D pair is larger than 25m, and distance between each D2D pair and the CUE whose uplink resource is reused is within $300 \mathrm{~m}$.

Table 2. Average EE Ratio.

\begin{tabular}{|c|c|c|c|c|}
\hline & Type 1 & Type 2 & Type 3 & Type 4 \\
\hline $\mathrm{N}=5$ & 3.24 & 2.95 & 2.4 & 1.72 \\
\hline $\mathrm{N}=10$ & 3.24 & 2.94 & 2.69 & 1.79 \\
\hline $\mathrm{N}=15$ & 3.21 & 2.99 & 1.96 & 1.19 \\
\hline $\mathrm{N}=20$ & 3.02 & 2.99 & 1.74 & 1.22 \\
\hline $\mathrm{N}=25$ & 3.12 & 2.81 & 1.77 & 1.19 \\
\hline
\end{tabular}

Table 2 shows the ratio of the average EE of our algorithm to the average EE without optimization. figure 3 shows the average EE of our algorithm in 4 types of scenarios. Results obviously present that the average EE in type 1 is the highest. That is because D2D 
cooperators are close to D2D requesters, which increases energy efficiencies, and CUEs whose resources are reused are far from those D2D pairs, which reduces the interferences between D2Ds and CUEs. In contrast, distant D2D pairs decrease energy efficiencies and closer CUEs cause more interference to D2D communications. Therefore, average EE of Type 4 is the lowest.

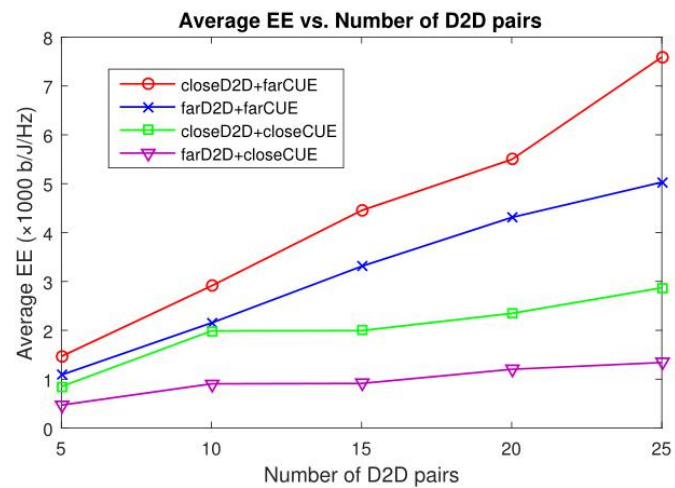

Fig. 3. Average EE vs. Number of D2D pairs.

\section{Conclusion}

In this paper, we investigated energy efficient cooperative D2D communications underlaying LTE-A networks. We proposed a cooperative D2D communication method in data forwarding, and formulated an optimization problem to maximize energy efficiency introduced by D2D communications. Simulation results show that average EE of our algorithm is much higher than that without optimization. In addition, we compare our algorithm in four types of scenarios to analyze how average EE is affected by the distances of D2D pairs and distances from D2D pairs to CUEs whose resources are reused by those D2D pairs. Results show that close distances between D2D pairs and far distance between

CUEs and D2D pairs achieve highest average EE.

\section{References}

1. Y. Chia, K. Doppler, C.B. Ribeiro, O. Tirkkonen, "Resource sharing optimization for device-to-device communication underlaying cellular networks", IEEE Trans. Wirel. Commun.,10 (8), pp. 27522763 (2011).

2. R. Yin, G. Yu, C. Zhong, and Z. Zhang, "Distributed resource allocation for D2D communication underlaying cellular networks", Proc. IEEE ICC, pp. 138143 (2013).

3. N. Golrezaei, P. Mansourifard, A.F. Molisch, A.G. Dimakis, "Base-station assisted device-to-device communications for high-throughput wireless video networks", IEEE Trans. Wirel. Commun.,13 (7), pp. 36653676 (2014).

4. X. Zhang, Z. Zheng, Q. Shen, J. Liu, X. Shen, L. Xie, "Optimizing network sustainability and efficiency in green cellular networks", IEEE Trans. Wirel. Commun.,13 (2), pp. 11291139 (2014).

5. D. Wu, J. Wang, "Energy-Efficient Resource Sharing for Mobile Device-to-Device Multimedia Communications", IEEE Transcations on Vechicular Technology, 63 (5), pp. 2093-2103, Jun. (2014).

6. H. Chen, D. Wu, Y. Cai, “Coalition Formation Game for Green Resource Management in D2D Communications", IEEE Communications Letters, 18 (8), pp. 1395-1398, Agu. (2014). 